\title{
Implantação da Sistematização da Assistência de Enfermagem como meio de qualificação da assistência ao idoso
}

\author{
Vanusa Maria Piccinini", Arlete Eli Kunz da Costa**, Luís Felipe Pissaia ${ }^{* *}$
}

\section{Resumo}

A Sistematização da Assistência de Enfermagem é uma metodologia de trabalho baseada em conhecimentos científicos, que oferece ao enfermeiro instrumentos de intervenção efetivos, neste caso, à população idosa. O objetivo deste estudo é descrever o processo de implantação da Sistematização da Assistência de Enfermagem como meio de qualificação da assistência ao idoso na Atenção Primária à Saúde em um município do Vale do Taquari, RS, Brasil. Trata-se de uma pesquisa descritiva e exploratória, com abordagem qualitativa. Os sujeitos de pesquisa são onze profissionais de enfermagem que atuam na Atenção Primária à Saúde de um município do Vale do Taquari, RS, Brasil. Os resultados evidenciam que os profissionais acreditam na importância de sistematizar o cuidado como meio de qualificação da assistência aos idosos. No entanto, foram evidenciados alguns entraves à implantação, como a falta de capacitação profissional e de conhecimento dos gestores sobre sua importância. A investigação aponta ainda o au- mento da demanda assistencial aos idosos, o que dificulta a prestação de cuidados efetivos pelas equipes, devido ao déficit de profissionais. Considera-se o estudo limitado, pois poucas pesquisas relacionadas à temática foram realizadas até o momento. No entanto, verificou-se que os profissionais têm responsabilidade ao assistir seus pacientes, oferendo uma assistência de qualidade, resolutiva e efetiva.

Palavras-chave: Sistematização da Assistência de Enfermagem. Equipe de enfermagem. Idoso.

\section{Introdução}

O aumento da expectativa de vida da população mundial pode ser considerado uma das maiores façanhas da humanidade, resultado de um esforço contínuo em combater os agentes que causam a falência do nosso organismo (ANDRADE

* Acadêmica do curso de Enfermagem do Centro Universitário Univates, Lajeado, RS, Brasil. E-mail: nusa@ universo.univates.br

** Enfermeira. Mestre em Desenvolvimento Regional. Doutora em Ambiente e Desenvolvimento. Docente do Centro de Ciências Biológicas e da Saúde do Centro Universitário Univates, Lajeado, RS, Brasil. E-mail: arlete.costa@univates.br

**** Enfermeiro. Mestrando em Ensino do Centro Universitário Univates, Lajeado, RS, Brasil. Endereço de correspondência: Av. Avelino Talini, 171, Prédio 11, Sala 216, Bairro Universitário, Lajeado, RS. CEP: 95914-014. E-mail: lpissaia@universo.univates.br

$\rightarrow$ http://dx.doi.org/10.5335/rbceh.v14i3.6631

Recebido em: 20.12.2016. Aceito em: 03.04.2018. 
et al., 2017). Se, por um lado, o envelhecimento é considerado uma vitória, por outro, se lança como um grande desafio, principalmente para as nossas estruturas sociais e econômicas, que, conforme cita Dias, Gama e Tavares (2017), estão despreparadas para assumir a demanda crescente de idosos.

De acordo com o Instituto Brasileiro de Geografia e de Estatística (IBGE), a pirâmide populacional brasileira demonstra mudanças significativas no crescimento da população idosa, pois o número de pessoas com 60 anos ou mais de idade teve um aumento superior a cinco milhões entre 1995 e 2005. Seguindo as projeções, em 2050, haverá um total de 34,3 milhões de idosos (IBGE, 2013). Machado et al. (2017) explicam essa transformação da pirâmide como decorrente da queda nas taxas de fecundidade e de mortalidade nas últimas décadas e do consequente aumento da expectativa de vida, além de mudanças sociais e ambientais no perfil demográfico, associado à diminuição da desigualdade social.

O envelhecimento humano é considerado um processo normal e natural; isto é, não significa presença de doença. Contudo, envelhecer com saúde depende de alguns fatores relacionados ao próprio indivíduo, principalmente às escolhas sobre os hábitos de vida. Além disso, conforme Ferreira et al. (2017), a atenção ao idoso é ampla, no sentido de integrar os órgãos públicos e da sociedade em geral, com o objetivo de promover ações que preservem a saúde e o bem-estar físico, mental e social e propiciem con- forto e melhoria na qualidade de vida das pessoas.

Assim, a área da saúde torna-se um terreno fértil para a propagação de iniciativas que estimulem e apoiem a assistência efetiva e igualitária à população na terceira idade, conforme Marandini, Silva e Abreu (2017), utilizando ferramentas de intervenção que fomentem o exercício de mudança social. Sob esse limiar de ação, Ferreira et al. (2017) citam a presença da equipe multiprofissional para atuar na linha de frente da Atenção Primária à Saúde (APS), comprometida com as necessidades do ser humano ao longo de todo o processo de viver e de morrer, incluindo a velhice, fase ainda tão marcada por preconceitos e tabus, estendendo-se os laços à família e à comunidade.

Nesse contexto, Machado et al. (2017) sugerem que a APS está dinamicamente envolvida com as transformações na sociedade, representada por profissionais que buscam mudanças e adequações na maneira de organizar o serviço e de atender a novas demandas sociais que se apresentam atualmente. Entre os profissionais apoiadores, Dias, Gama e Tavares (2017) destacam a importância da equipe de enfermagem, que, constituída por indivíduos sociais, desenvolve o senso crítico e reflexivo construído por meio do conhecimento científico e da aproximação com a comunidade.

Tendo como alicerce esses conceitos, tornou-se cada vez mais contundente a iniciativa de qualificar a assistência oferecida à população idosa, compreendendo como metodologia de trabalho a 
Sistematização da Assistência de Enfermagem (SAE), que, conforme Oliveira et al. (2017), compreende conceitos próprios da área, mas incentiva a interdisciplinaridade. Tal afirmativa é almejada na comunicação interpessoal, facilitada pelo processo da SAE na APS, aproximando o indivíduo da equipe, por meio do conhecimento intrínseco e sistematizado da realidade na qual ele está inserido (TANNURE; PINHEIRO, 2013).

Nesse sentido, este estudo objetiva descrever o processo de implantação da SAE como meio de qualificação da assistência ao idoso na APS em um município do Vale do Taquari, RS, Brasil.

\section{Materiais e métodos}

Trata-se de um estudo descritivo e exploratório, com abordagem qualitativa. Os participantes da pesquisa são onze enfermeiros que atuam nas cinco unidades de APS de um município do Vale do Taquari, RS, Brasil.

Os participantes foram identificados pelos pesquisadores por meio de carta de anuência encaminhada à gestão municipal em saúde do referido município, que informou os contatos dos onze enfermeiros atuantes na APS. De posse das informações necessárias, os pesquisadores entraram em contato com os profissionais, para, neste momento, explicar os objetivos do estudo e os critérios éticos norteadores do projeto de pesquisa. Os onze profissionais atuantes aceitaram participar da pesquisa, sendo, então, agendada uma data e definido o local para a realização da entrevista.
Os pesquisadores visitaram os locais de trabalho dos profissionais e realizaram as entrevistas durante os meses de agosto e setembro de 2016. As entrevistas ocorreram de forma individual, em espaço que oferecia confidencialidade ao diálogo. $\mathrm{Na}$ ocasião, foram reforçados os objetivos do estudo e os critérios éticos, norteadores de todo o projeto de pesquisa. Logo após, foram lidos e assinados os termos de consentimento livre e esclarecido em duas vias de igual teor.

Os diálogos tiveram duração média de trinta minutos. Foi aplicado questionário próprio, desenvolvido pelos pesquisadores, composto por questões abertas e semiestruturadas, que contemplaram o objetivo do estudo. As entrevistas foram gravadas em áudios que, posteriormente, foram transcritos e analisados por pontos focais compatíveis, conforme prevê a análise de conteúdo de Bardin (2011).

Visando à proteção ética dos sujeitos, realizou-se a troca dos nomes próprios pela abreviatura do substantivo enfermeiro, "Enf.", seguido de números ordinais de 1 a 11, sorteados aleatoriamente. Acordou-se que os dados levantados pela pesquisa seriam apresentados formalmente pelos pesquisadores em formato de trabalhos científicos à comunidade de estudo.

Este artigo faz parte do projeto de pesquisa A Equipe Multiprofissional e o Gerenciamento dos Investimentos Públicos Aplicados na Saúde dos Idosos em um Município da Região Noroeste do Estado do Rio Grande do Sul. Para a realização do estudo, os pesquisadores obtiveram aprovação do projeto de 
pesquisa pela Pró-Reitoria de Ensino e pelo Comitê de Ética em Pesquisa da Centro Universitário Univates por meio da CAAE nº 11992112.8.0000.5310. Observaram-se ainda os critérios éticos previstos pela Resolução no 466, de 2012, do Conselho Nacional de Saúde, relativa a pesquisas com seres humanos.

\section{Resultados}

Analisando as informações coletadas, verificou-se que os enfermeiros acreditam na eficácia da SAE no contexto da assistência ao idoso. Constatou-se, ainda, a sensatez dos profissionais ao se referirem à qualificação trazida pela $\mathrm{SAE}$ ao serviço, conferindo qualidade à assistência, conforme ilustram as declarações a seguir:

Para mim é a organização e realização do processo de enfermagem com a visão por completo do paciente, principalmente o idoso, proporcionar cuidados de boa qualidade (Enf. 01).

É uma atividade cabível ao profissional enfermeiro oferecer ao paciente uma melhor qualidade no atendimento ao paciente (Enf. 05).

Quando questionados sobre o processo de implantação da SAE em seus locais de trabalho, os sujeitos referem que, na maioria dos casos, algumas etapas do Processo de Enfermagem (PE) já vinham sendo utilizadas há mais tempo para a população em geral, como forma de registro das atividades no prontuário do indivíduo, o que foi um facilitador para a implantação. No entanto, alguns profissionais também relataram que, apesar das etapas em uso durante a prestação da assistência, é necessário oferecer educação continuada, com a finalidade de capacitar e de incentivar a utilização da SAE na APS do município, conforme os depoimentos:

Quando cheguei nesta unidade já existia um processo onde realizamos a SAE em partes, durante $o$ acolhimento, conseguimos descrever o histórico do paciente, realizar o exame físico e em seguida uma breve evolução de enfermagem no prontuário da unidade. Porém é preciso reavaliar, corrigir e implementar a cada dia. Entretanto, a implantação da SAE depende de cada enfermeiro (Enf. 01).

Infelizmente há uma grande dificuldade de implantação da SAE nas unidades de saúde do nosso município, devido a falta de incentivo e capacitação profissional (Enf. 03).

Por meio das observações realizadas em campo e das informações coletadas, verificaram-se alguns empecilhos no que diz respeito à implantação da SAE na APS do município. Outra questão levantada é a crescente demanda por assistência à pessoa idosa, que exige da equipe uma estrutura de recursos humanos indisponível no local, o que acarreta dificuldades para realizar a SAE durante os atendimentos, conforme os relatos:

A quantidade de atividades que o enfermeiro assume em relação à supervisão dos usuários idosos, principalmente os doentes crônicos (Enf. 01).

Devido a grande demanda da população idosa e falta de profissionais para atender, faltando um dimensionamento adequado, falta de equipamentos e materiais de trabalho (Enf. 03). 
Outras questões que apareceram fortemente como empecilhos na implantação da SAE são a falta de preparo das equipes na compreensão do processo de gestão e a falta de estímulo e de incentivo, por parte do Poder Público, em qualificar a assistência realizada na APS. Os entrevistados consideram que a falta de preparo desestimula a utilização da SAE, devido ao desconhecimento de suas atribuições e benefícios, principalmente com a população idosa, conforme as declarações:

Falta de preparo das equipes em fazer a SAE com toda a população e ainda mais com os idosos (Enf. 03).

Equipes despreparadas, sem conhecimento, comprometimento, conhecimento específico e falta de tempo ou disponibilidade da equipe (Enf. 04).

Questionados a respeito da interação das equipes com os gestores municipais, principalmente os da saúde, no que diz respeito ao uso da $\mathrm{SAE}$, os sujeitos relatam que não há uma comunicação eficiente em geral e, muito menos, em relação à importância da SAE. Comentou-se que a implantação da SAE não está sendo exigida por parte dos gestores, o que transmite certa insegurança e um descrédito em relação à assistência oferecida nos espaços de saúde, conforme os depoimentos:

No momento, não vejo motivação, e nem interação pela gerência. Não existe muita cobrança por parte deles. Mas acredito que, se soubessem da importância, poderiam ajudar para que a SAE fosse implantada (Enf. 02).
Não percebo motivação, não está sendo exigido e nem cobrado. As pessoas não aderem às mudanças quando não são estimuladas, pois nós tentamos fazer, e eles não ajudam, e desanimamos (Enf. 04).

No que diz respeito à utilização da SAE como meio de apoiar a assistência ao idoso, os profissionais relatam que muito poderia ser feito para a qualificação do acompanhamento oferecido a esta população. São relacionados os benefícios que um conhecimento ampliado sobre a realidade do idoso poderia trazer à comunidade, principalmente quando se utiliza a SAE como ferramenta de intervenção individualizada, agindo in loco, onde está o problema, conforme os relatos:

Para o idoso, a SAE é muito importante, pois a equipe poderia ajudá-lo num todo, buscando a comunicação e informação de qualidade, tendo em vista um olhar individualizado e a real situação em que se encontra o paciente no momento e no seu vínculo familiar (Enf. 02).

Um atendimento individualizado e com meIhor qualidade a cada paciente. Conhecer o paciente como um todo. O contexto social em que está inserido, meio em que vive, doença e família (Enf. 05).

A SAE ainda foi abordada pelos profissionais como instrumento assistencialista domiciliar, isto é, pode ser utilizada na atenção e no acompanhamento domiciliar de idosos. A realização das etapas da SAE confere um conhecimento peculiar acerca da história de vida do indivíduo e do seu contexto, fomentando a utilização destas informações por meio de um projeto terapêutico singular, específico para as necessidades daquele domicílio, conforme as declarações: 
Quando realizo visita domiciliar ao idoso, procuro dar esse olhar mais especial a eles, pois muitos se encontram fragilizados e precisando de uma atenção maior por parte da enfermagem com a SAE (Enf. 02).

Acredito que dá para fazer a SAE sim; na visita domiciliar, é possível identificar o ambiente em que esse idoso está inserido, realizar um histórico de enfermagem, o exame físico, diagnóstico de enfermagem e evolução de enfermagem (Enf. 05).

Os entrevistados finalizaram as entrevistas presumindo que a SAE poderia auxiliar em diferentes contextos de atuação, principalmente para qualificar a assistência e oferecer um cuidado efetivo e resolutivo aos idosos. Indicando a SAE como forma de criação de vínculo terapêutico e de observação integral de cada caso particular, atentando, ainda, para os benefícios na gestão da própria unidade de saúde, são ilustrativas as declarações a seguir:

Com a SAE, poderíamos dar mais atenção ao idoso, um atendimento humanizado e com melhor qualidade. Poderia haver mais respeito e organização no trabalho em equipe (Enf. 02).

$\mathrm{O}$ atendimento ao idoso poderia ser mais organizado, aperfeiçoar a capacidade de solucionar problemas, assim, fortalecendo o vínculo com os pacientes (Enf. 04).

\section{Discussão}

Colaborando com os dados dos estudos de Oliveira et al. (2017) sobre a implementação da SAE, reiteramos sua importância enquanto uma metodologia de organização, de planejamento e de execução de ações sistematizadas, que são realizadas pela equipe durante $o$ período em que o cliente se encontra sob a assistência de enfermagem, sendo guiada pelo $\mathrm{PE}$, melhorando o atendimento oferecido. Nesse sentido, os estudos de Tannure e Pinheiro (2013) nos incentivam a realizar uma reflexão sobre a SAE enquanto uma metodologia científica que vem sendo cada vez mais implementada no exercício de assistência à população, com o objetivo de garantir maior segurança e qualidade na assistência e na autonomia da equipe de enfermagem; sobretudo, colaborando com os achados, a sistematização confere qualificação à assistência prestada à população.

Portanto, a SAE é uma atividade privativa do enfermeiro. Os relatos estão em consonância com a Lei do Exercício Profissional no ${ }^{\circ} .498$ (CONSELHO FEDERAL DE ENFERMAGEM, 1986) e a Resolução do Conselho Federal de Enfermagem (Cofen) no 358 (CONSELHO FEDERAL DE ENFERMAGEM, 2009). Nesses documentos, é reforçado que sua implantação deve ser realizada em toda a instituição de saúde pública e privada, colaborando com os achados e ainda em acordo com a necessidade de utilizar o PE como meio de implantação (COFEN, 1986). Para Marandini, Silva e Abreu (2017), a SAE organiza o trabalho profissional, podendo contribuir para a promoção, a prevenção, a recuperação e a reabilitação da saúde do indivíduo, da família e da comunidade em qualquer esfera de atenção à saúde, sendo constatado nas entrevistas 
o diferencial que a implantação da SAE traz para o serviço, principalmente com a qualificação dos processos.

A SAE é reconhecida pelos profissionais de enfermagem como marco a ser institucionalizado nos serviços de saúde, principalmente após à Resolução do Cofen no 272/02, que dispõe sobre a SAE nas instituições de saúde no Brasil e determina que sua implementação deva ocorrer em todas as instituições de saúde, tanto públicas quanto privadas (COFEN, 2002). Nesse sentido, um levantamento bibliográfico realizado por Andrade et al. (2017), com estudos publicados entre 2000 e 2010, verificou que os obstáculos mais frequentemente mencionados para a implementação da SAE envolvem: número reduzido de profissionais de enfermagem e, consequentemente, sobrecarga de trabalho; falta de capacitação da equipe; falta de comunicação; resistência por parte dos profissionais, diretamente relacionada ao desconhecimento do tema, devido à falta de liderança e de educação continuada; falta de disposição e de disponibilidade para enfrentar mudanças e para reorganizar o trabalho de enfermagem; baixo investimento das instituições em qualificação de recursos humanos. Este estudo colabora diretamente com os achados da pesquisa, compartilhando as dificuldades na implantação da SAE.

Ainda sobre a equipe, Marandini, Silva e Abreu (2017) trazem a capacitação como importante meio para o desenvolvimento do agir profissional de enfermagem, uma vez que confere segurança na tomada de decisões relacionadas ao paciente, à equipe e às atividades administrativas da unidade, incorporando, assim, as similaridades com os relatos dos entrevistados sobre a importância da educação continuada em seu contexto de trabalho. A segurança profissional descrita em muitos estudos e problematizada durante a aplicação dos questionários se refere à liderança profissional perante a equipe e seu incentivo aos demais, o que sem dúvida colabora com o estudo realizado por Clares, Borges e Freitas (2017), com oito enfermeiras do Hospital São Paulo, SP, em 2002, aparecendo como um reflexo na equipe de enfermagem, que percebe o enfermeiro como um condutor dos processos assistenciais.

Estudos nacionais, como o realizado por Guedes et al. (2017), relacionam alguns problemas que podem levar os enfermeiros a não sistematizar a assistência e colaboram com os achados do presente estudo, como, por exemplo: formação acadêmica inadequada; falta de comprometimento dos enfermeiros; perfil e postura inadequados; desinteresse; recursos financeiros insuficientes para o aprimoramento profissional; problemas de relacionamento interpessoal; falta de liderança e de organização; falhas na estrutura administrativa da instituição.

Para Ferreira et al. (2017), a preocupação com a qualidade na área da saúde é assunto antigo; entretanto, as propostas de sistematização e de organização dos serviços são recentes no ciclo do pensamento administrativo dos ges- 
tores em saúde, o que colabora para as dificuldades de aceitação dos gestores na implantação da SAE, conforme verificado nos relatos. $\mathrm{O}$ autor ainda sugere que, devido à multiplicidade das demandas, das necessidades e das especificidades do indivíduo e do coletivo, exige-se que os gestores busquem estimular o desenvolvimento e o alcance de capacidades e atitudes inovadoras e criativas (FERREIRA et al., 2017).

Ainda, no levantamento bibliográfico realizado por Andrade et al. (2017), apontou-se a SAE como um instrumento relevante para o avanço do desempenho institucional, até mesmo necessário à efetivação dos princípios e valores que norteiam a atual política de saúde, uma vez que intervém de maneira eficiente e competente no complexo sistema que configura os serviços de saúde, bem como, no atual cenário de envelhecimento populacional, interage diretamente com o presente estudo, sugerindo uma alternativa ao aumento da demanda por assistência aos idosos.

A necessidade de atendimento da pessoa idosa é firmada em 1999, quando o Ministério da Saúde anuncia a Portaria $\mathrm{n}^{\mathrm{o}}$ 1.395/99, Política Nacional de Saúde do Idoso (PNSI), que determina que os órgãos e entidades do Ministério da Saúde relacionados ao tema promovam a elaboração ou a readequação de planos, projetos e atividades em conformidade com as diretrizes e responsabilidades estabelecidas (GRANGEIRO et al., 2018). A referida portaria tem como diretrizes: a promoção do envelhecimento saudá- vel; a manutenção e a reabilitação da capacidade funcional; a assistência às necessidades de saúde; a capacitação de recursos humanos especializados; o apoio ao desenvolvimento de cuidados informais; o apoio a estudos e pesquisas. Quanto aos cuidados prestados aos idosos, a PNSI orienta o processo contínuo de avaliações, que deve acompanhar desenvolvimento e, conforme Clares, Borges e Freitas (2017), abrir portas para a implementação da SAE.

Nesse contexto, para Grangeiro et al. (2018), a APS é um espaço assistencial enriquecedor para a implementação da SAE, visto que proporciona atenção especial ao idoso, bem como desempenha participação ativa na melhoria da qualidade de vida, com medidas de promoção, proteção, identificação precoce de agravos, intervenção e medidas de reabilitação, voltadas a evitar a exclusão do idoso do convívio familiar e social. Nesse sentido, Moraes (2017) instrumentaliza a necessidade de educação continuada dos profissionais dos serviços, para que: sejam capazes de planejar e de programar ações; estejam preparados para lidar com as questões do processo de envelhecimento; abordem adequadamente a pessoa idosa; e busquem sempre o máximo de autonomia dos usuários.

Para tanto, a crescente demanda por conhecimento, que vai sendo agregado aos serviços na área da enfermagem como alternativa de aperfeiçoamento e de atualização dos profissionais de enfermagem para realizar seu trabalho com qualidade e de maneira humaniza- 
da, tem como foco o estudo e as práticas relacionados ao cuidado integral e individual, de acordo com Dias, Silva e Tavares (2017), o que colabora com a busca por qualificação profissional e assistencial predominante nos achados. $\mathrm{Na}$ era do conhecimento, Guedes et al. (2017) incitam que a implementação de um modelo e/ou de uma fórmula predeterminada de assistência não é garantia de maior qualidade na assistência em saúde, no entanto, com o estímulo e o reconhecimento dos enfermeiros, a implantação da SAE torna-se efetiva e resolutiva.

\section{Conclusão}

A SAE é um tema ainda pouco estudado na APS como forma de organização do trabalho e de qualificação da assistência, o que instigou ainda mais os autores a estudar o assunto. Contudo, verificou-se que a implantação da SAE oferece indicativos de benefícios ao idoso, principalmente por meio da qualificação da assistência oferecida pelos enfermeiros.

Os resultados demonstraram que a SAE exige do profissional muito mais do que capacidade técnica. Ou seja, é necessário aliar o conhecimento científico com a sensibilidade própria do profissional, a fim de aproximar o indivíduo da equipe. Nesse sentido, é necessário que o profissional invista em aperfeiçoamento, ampliando e aprofundando continuamente os saberes específicos de sua área de atuação.

Este estudo evidenciou que as principais dificuldades para a implementação da SAE dizem respeito à sobrecarga de trabalho e, ainda, ao número insuficiente de profissionais para a execução das atividades em geral e, especificamente, para 0 atendimento à população idosa. $\mathrm{O}$ desconhecimento dos gestores de saúde também é um empecilho na utilização da SAE, sendo necessária sua propagação como ferramenta eficaz de cuidado.

A qualificação da assistência vem sendo percebida pelas equipes como diferencial na atuação profissional com o uso da SAE; portanto, espera-se que a adesão a esta metodologia de trabalho cresça no atendimento à população idosa, a fim de instrumentalizar um cuidado efetivo e resolutivo. Considera-se que este estudo possa contribuir para a reflexão de práticas na saúde do idoso realizadas na área de APS, instigando os profissionais a buscar a qualificação dos processos de trabalho, considerando a SAE como facilitadora.

\section{Implantation of the Systematization of Nursing Assistance as a means of qualifying care for the elderly}

\section{Abstract}

The Systematization of Nursing Care is a work methodology based on scientific knowledge, which offers nurses effective intervention tools, in this case, to the elderly population. The objective of this study is to describe the process of implementation of Nursing Care Systematization as a means of qualifying care for the elderly in Primary Health Care in a municipality of Vale do Taquari, RS, Brazil. This is a descriptive and exploratory research with a qualitative 
approach. The research subjects are eleven nursing professionals who work in the Primary Health Care of a municipality of Vale do Taquari, RS, Brazil. The results showed that professionals believe in the importance of systematizing care as a means of qualifying care for the elderly. However, some impediments to the implantation were evidenced, such as the lack of professional qualification and the knowledge of managers about their importance. The research also points out the increase in the care demand for the elderly, which makes it difficult to provide effective care for the teams due to the professional deficit. The study is considered limited, since few studies related to the subject have been carried out to date. However, it was verified that the professionals have the responsibility to assist their patients, offering a quality assistance, resolutive and effective.

Keywords: Elderly. Nursing care systematization. Nursing team.

\section{Referências}

ANDRADE, I. A. et al. Capacitação em saúde do idoso para os agentes comunitário de saúde da UBS Citrolândia em Betim-MG: relato de uma prática intervencionista. Sinapse Múltipla, Betim, MG, v. 6, n. 2, p. 267-272, jul./dez. 2017.

CLARES, J. W. B.; BORGES, C. L.; FREITAS, M. C. D. Idosos institucionalizados: proposta educativa na perspectiva freireana. Revista de enfermagem on-line, Universidade Federal de Pernambuco, v. 11, n. 2, p. 10731077, abr./jun. 2017.

CONSELHO FEDERAL DE ENFERMAGEM. Lei $n^{\circ} 7.498$, de 25 de junho de 1986. Disponível em: <http://www.cofen.gov.br/ lei-n-749886-de-25-de-junho-de-1986_4161. html>. Acesso em: 10 maio 2016.
CONSELHO FEDERAL DE ENFERMAGEM. Resolução $n^{\circ} 272$, de 27 de agosto de 2002. Disponível em: <http://www. scielo.br/scielo.php?script=sci_arttext\&pi$\mathrm{d}=\mathrm{S} 1414-81452007000300010>$. Acesso em: 11 abr. 2016.

DIAS, F. A.; GAMA, Z. A.; TAVARES, D. M. Atenção primária à saúde do idoso: modelo conceitual de enfermagem. Cogitare Enfermagem, Curitiba, v. 22, n. 3, p. 1-11, jul./ set. 2017.

FERREIRA, L. V. et al. Busca do autocuidado por idosos na rede de atenção à saúde. Revista Contexto \& Saúde, Ijuí, v. 17, n. 32, p. 46-54, jun. 2017.

GRANGEIRO, A. F. B. et al. Atividade física e cognição para promoção da saúde de idosos: um relato de experiência no norte do Tocantins. Capim Dourado: Diálogos em Extensão, Palmas, v. 1, n. 1, p. 05-12, jan. 2018.

GUEDES, M. B. O. G. et al. Apoio social e o cuidado integral à saúde do idoso. Physis: Revista de Saúde Coletiva, São Paulo, v. 27, p. 1185-1204, out./dez. 2017.

INSTITUTO BRASILEIRO DE GEOGRAFIA E ESTATÍSTICA - IBGE. Síntese de Indicadores Sociais: uma análise das condições de vida da população brasileira, 2013. Disponível em: <http://biblioteca.ibge.gov.br/ visualizacao/livros/liv66777.pdf>. Acesso em: 08 maio 2016.

MACHADO, W. D. et al. Idosos com doenças crônicas não transmissíveis: um estudo em grupos de convivência. Revista Ciência \& Saberes-Facema, São Luís, v. 3, n. 2, p. 445451, abr./jun. 2017.

MARANDINI, B. A. N.; SILVA, B. T. D.; ABREU, D. P. G. Avaliação da capacidade funcional de idosos: atividade das equipes da Estratégia de Saúde da Família. Revista de Pesquisa: Cuidado é Fundamental, Rio de janeiro, v. 9, n. 4, p. 1087-1093, out./ dez. 2017. 
MORAES, E. N. Idosos frágeis e a gestão integral da saúde centrada no idoso e na família. Revista Brasileira de Geriatria e Gerontologia, São Paulo, v. 20, n. 3, p. 307308, maio/jun. 2017.

OLIVEIRA, M. S. N. et al. Autocuidado de idosos diagnosticados com hipertensão arterial e/ou diabetes mellitus. Revista de Enfermagem da Universidade Federal de Santa Maria, Santa Maria, RS, v. 7, n. 3, p. 490-503, jul./set. 2017.

TANNURE, M. C.; PINHEIRO, A. M. SAE - Sistematização da Assistência de Enfermagem: guia prático. Rio de Janeiro: Guanabara Koogan, 2013. 